\title{
Modelling of Dielectric Barrier Discharge Plasma Actuators for Direct Numerical Simulations
}

\author{
Timothy Brauner * and Sylvain Laizet ${ }^{\dagger}$ \\ Imperial College London, London, SW7 2AZ, UK \\ Nicolas Benard $\ddagger$ and Eric Moreau ${ }^{\S}$ \\ Institut PPRIME - CNRS - University of Poitiers ENSMA, 86962, Futuroscope, France
}

\begin{abstract}
In recent years the development of devices known as plasma actuators has advanced the promise of controlling flows in new ways that increase lift, reduce drag and improve aerodynamic efficiencies; advances that may lead to safer, more efficient and quieter aircraft. The large number of parameters (location of the actuator, orientation, size, relative placement of the embedded and exposed electrodes, materials, applied voltage, frequency) affecting the performance of plasma actuators makes their development, testing and optimisation a very complicated task. Several approaches have been proposed for developing numerical models for plasma actuators. The discharge can be modelled by physics-based kinetic methods based on first principles, by semi-empirical phenomenological approaches and by PIV-based methods where the discharge is replaced by a steady-state body force. The latter approach receives a recent interest for its easy implementation in RANS and U-RANS solvers. Here, a forcing term extracted from experiments is implemented into our high-order Navier-Stokes solver (DNS) in order to evaluate its robustness and ability to mimic the effects of a surface dielectric barrier discharge. This experimental forcing term is compared to the numerical forcing term developed by Suzen \& Huang $(1,2)$ with an emphasis on the importance of the wall-normal component of each model.
\end{abstract}

\section{Nomenclature}

$V / m$

$\varepsilon_{0} \quad$ Permittivity of Free Space, $\mathrm{C}^{2} / \mathrm{Nm}^{2}$

$\varepsilon_{r} \quad$ Relative Permittivity

$\vec{f}_{B} \quad$ Body Force Vector, $N / m^{3}$

$f_{x} \quad$ Wall-parallel Component of Body Force Vector, $N / \mathrm{m}^{3}$

$f_{y} \quad$ Wall-normal Component of Body Force Vector, $N / m^{3}$

$\rho_{c} \quad$ Charge Density, $C / m^{3}$

$\Phi \quad$ Electric Potential

$\phi \quad$ Electric Potential due to external electric field, $V$

$\varphi \quad$ Electric Potential due to net charge density, $V$

$\lambda_{D} \quad$ Debye Length, $m$

$f_{A C}$ Frequency, $\mathrm{Hz}$

$P \quad$ Pressure, $\mathrm{Pa}$

$\rho \quad$ Density, $\mathrm{kg} / \mathrm{m}^{3}$

$\nu \quad$ Kinematic Viscosity $\mathrm{m}^{2} / \mathrm{s}$

U Velocity, $\mathrm{m} / \mathrm{s}$

\footnotetext{
*Research Assistant, Department of Aeronautics, Imperial College London, UK.

${ }^{\dagger}$ Lecturer, Department of Aeronautics, Imperial College London, UK.

¥Associate Professor, Institut P', University of Poitiers, France.

$\S$ Professor, Institut P', University of Poitiers, France.
} 


\section{Introduction}

In recent years there has been considerable interest in the use of dielectric barrier discharges (DBD) plasma actuators for flow control $(3,4,5)$. Some of the reasons for the popularity of these actuators are their special features that include being fully electric with no moving parts, a fast time response and highfrequency forcing for unsteady applications, a very low mass which is especially important in applications with high g-loads, a low power consumption and a low protrusion in the flow with virtually no drag penalty when the active control system is turned off. As shown in figure 1, a typical DBD plasma actuator consists of two electrodes (dark blue), one exposed to the ambient fluid and the other covered by a dielectric material (grey). The two electrodes are supplied with an A.C. voltage which causes a surface discharge. This ionized gas results in a body force which exchanges momentum with the ambient neutrally charged fluid. In a quiescent fluid, a DBD plasma actuator creates an induced flow towards the edge of the exposed electrode in the direction of the covered electrode and a jetting of the flow towards the far edge of the covered electrode (6), as seen in figure 1 (left). For a boundary layer, the plasma actuator adds momentum to the flow with an acceleration of the boundary layer, as seen in figure 1 (right).

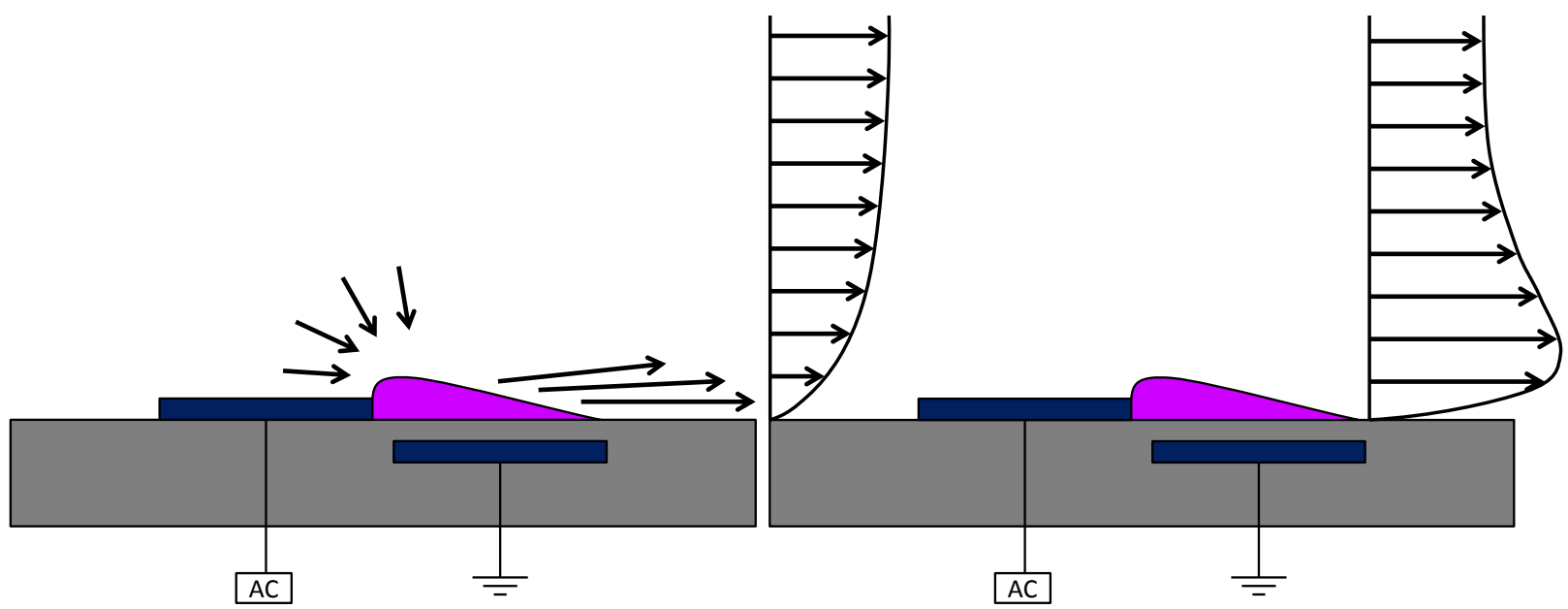

Figure 1. Sketch of the effect of a dielectric barrier discharge (DBD) plasma actuator in a quiescent ambient fluid (left) and in a boundary layer (right).

Computational studies of plasma flow control have been limited in comparison to the vast number of experimental studies. To include the effect of the actuators in numerical simulations, the spatial distribution and the magnitude of the body force have to be known. Attempts at modelling plasma actuators can be divided into two groups: first-principle based models and simplified (phenomenological) models. The first-principle based models use a fundamental approach to model the physical mechanisms of a plasma actuator. They require the solution of complex transport equations for both charged and neutral species, a Poisson equation for the electric field and the Navier-Stokes equations. Three-dimensional simulations based on first principle were performed with a non-uniform body force in the spanwise direction and the results were compared with two-dimensional simulations $(7,8)$. It was shown that two-dimensional simulations underestimate the chordwise extension of the force field and the force amplitude due to weaker electric field concentration in the two-dimensional structures of the plasma. Those models require very long computational times, up to one order of magnitude more than the simplified models $(9,10,11,12)$. These elegant approaches still have room for improvement as plasma discharges involve many kinetics as recombination, ionization and attachment. In the case of dielectric barrier discharge the model should account for the charging of the dielectric surface caused by the propagation of the three-dimensional ionized channels and for nanosecond time scales (lifetime of the ionized filaments).

Simplified models attempt to capture the ionization effects of the plasma actuator without directly modelling the species transport equations. Those models are based on the assumption that the plasma formation and fluid flow response can be decoupled due to the large disparities in the characteristic velocities associated with each process (13). The most popular of those models are the Orlov model (14), the Shyy model $(15)$ and the Suzen \& Huang model $(1,2)$ because of their relative simplicity and ability to mimic 
the time-averaged effects of a DBD actuator on the ambient fluid. Those three models have been tested in various flow configurations: boundary-layer separation control for the Orlov model (14), turbine blades $(1,2)$, channel flow (16) and tandem of cylinders (17) for the Suzen \& Huang model and channel flow (18) and transition control around an airfoil (19) for the Shyy model. These models have two major drawbacks. Firstly, some parameters of the models correspond to physical quantities for which exact values are not well defined, leading to the use of corrections to agree with a given set of experimental results. Secondly, they are time-independent and the unsteadiness of the plasma actuators cannot be taken into account when even low amplitude fluctuations in the produced flow (also referenced as electric wind) can trigger flow instabilities and can have a drastic influence on the control effect. Recent investigations have been made at reproducing the non-stationary character of the produced flow while using a modified Suzen \& Huang model that is originally time-independent. In these studies $(20,21,22)$, the Suzen \& Huang model is modulated by a sinusoidal wave in order to reproduce the influence of the driving AC frequency but the model is also further modulated in burst mode in order to achieve low-frequency periodic perturbations as performed in experiments. This advanced modelling has been validated for quiescent fluid conditions by a comparison with experiments in 20 but the method has also been implemented for manipulating a stalled NACA0015 aerofoil $(21,22)$ and the obtained numerical results confirm that the lift and drag coefficients can been significantly improved by using burst mode actuation.

In order to improve the quality and the reliability of the numerical models, some attempts have been made recently to determine the force distribution experimentally using advanced optical techniques such as particle image velocimetry (PIV) measurements $(23,24,25,26,27)$. The most straightforward strategy is based on the two-dimensional incompressible Navier-Stokes equations. Assuming that the pressure gradients are much smaller that the force components, they can be neglected and because all the other components of the Navier-Stokes equations can be computed from the experimental data, the force components can be evaluated fairly easily. It is also possible to evaluate the force components using the vorticity transport equation to eliminate the pressure terms, assuming that the force is dominated by one component only (it is usually assumed that the wall-normal component of the force is much smaller than the wall-parallel component), it can be calculated (23). Comparisons of the two approaches are given in $(26,28,29)$. Direct Numerical simulations (DNS) were performed very recently in 29 to investigate the quality of the approach based on the Navier-Stokes equations and the approach based on the vorticity transport equation. The authors used an empirical model designed to reproduce the velocity field obtained by 26 via a retroactive estimation of the volume force from experimental results. They observed that when the ambient fluid is not zero the pressure gradients may not be neglected for the Navier-Stokes approach. It was also shown in 30 that both approaches provide a more accurate estimate for distributions and magnitude of the modelled body force than the numerical phenomenological model of Shyy (15) and of Suzen \& Huang $(1,2)$. It should be noted that the authors in this study did not investigate the Orlov model (14).

The main objectives of the present study are to discuss the relevance of the Suzen \& Huang model $(1,2)$ in a context of Direct Numerical Simulations (DNS) and to compare it with an experimental forcing term obtained from PIV data via the Navier-Stokes approach. DNS are performed both with the numerical and experimental forcing and an attempt to simulate the starting vortex experiment of 31,32 is made. For the experimental forcing, the validity of the assumptions used to extract the forcing terms from the PIV data are investigated (Can the pressure gradients be neglected?). The effect of the wall-normal force component on the resulting flow field is also examined as it is very often assumed that this force component can be neglected (29). The paper is organized as follow: in the next section the method to obtain our experimental PIV-based model is described. Then the following section is dedicated to the implementation of the phenomenological Suzen \& Huang model in our high-order flow solver Incompact3d. Then the results section is divided in three sub-sections with first a validation of the numerical forcing terms via a comparisons with the original data of 2 , followed by an attempt to reproduce the experimental data of 31,32 for the formation and evolution of a starting vortex and by a detailed comparison between our experimental PIV-based model and the numerical phenomenological Suzen \& Huang model. The paper ends with a conclusion section.

\section{Experimental PIV-based model}

\section{A. Experimental set-up}

PIV measurements have been conducted for different voltages (from 12 up to $20 k V_{a m p}$ ) and frequencies (from $100 \mathrm{~Hz}$ to $2000 \mathrm{~Hz}$ ) in order to characterize the effect of a DBD plasma actuator in an ambient fluid 
at rest (33). The spatial distribution of the mean force resulting from the plasma actuator is evaluated based on the mean velocity field and inversion of the Navier-Stokes equations $(27,34)$. A detailed description of the method can be found in $(24,26,33)$. To summarize, it consists of measuring the mean induced flow produced at the center of the actuator $(z=0)$ thanks to a PIV acquisition system that is triggered by the applied voltage signal. The PIV system is composed of dual-head NdYAG laser (Evergreen Big Sky, Quantel), a CCD camera with $4920 \times 3280$ resolution (LX 16M, Lavision), a trigger unit and a multi-core PC running Davis 8 PIV software. The camera is equipped with a $105 \mathrm{~mm}$ lense to record a field of view of $40 \times 14 \mathrm{~mm}$ (spatial resolution of $8.1 \mu \mathrm{m}$ ) per pixel. The flow is seeded with droplets of dielectric oil (Ondina 919) with a particle diameter equal to $0.3 \mu \mathrm{m}$. The velocity fields are obtained by a cross correlation algorithm with adaptive multi-pass (final resolution of one vector every $65 \mu \mathrm{m}$ ). The time-averaged velocity field is obtained by recording 6,000 images for each configuration and the data processing is performed on a cluster of $60 C P U$ running at $3.6 \mathrm{GHz}$. In this work, we focus on one actuator which is made of a $10 \mathrm{~mm}$ air-exposed and a $20 \mathrm{~mm}$ grounded electrode (with an inter-electrode distance of $1 \mathrm{~mm}$ ), these two electrodes are placed on both side of a $3 \mathrm{~mm}$ thick PMMA plate acting as dielectric barrier.

\section{B. Force model by PIV-based approach}

The mean flow produced by the discharge obeys the incompressible two-dimensional Navier-Stokes equations. At each data point of the measured velocity field, the mean volume force $\mathbf{F}$ can be expressed as

$$
\mathbf{F}-\nabla p=\rho \mathbf{U} \cdot \nabla \mathbf{U}-\nu \nabla^{2} \mathbf{U}
$$

where $p$ is the pressure field, $\mathbf{U}$ is the mean velocity field while $\rho$ and $\nu$ are the gas density and gas dynamic viscosity, respectively. The right hand side of this equation can be evaluated at each measurement location from the velocity measurements. It is considered here that the pressure contribution is minor regarding the volume force produced by the discharge. This point will however be checked later on in this study. Subsequently, the left hand side approximates the volume force $\mathbf{F}$. One way to validate this approach consists of computing the body force thrust as the spatial integral of the volume force. Providing that the integration surface is well-adapted to the problem, a very good agreement was found between PIV-based indirect body force estimation and direct measurements by a force balance (33). This experimental forcing term can also be implemented in our high-order flow solver in order to check if it is possible to recover the time-average velocity fields obtained by the PIV measurements. The coupling with a DNS solver is also expected to validate or refute the used of PIV-based models extracted from the Navier-Stokes equations or at least provide some clarifications on the effect of discarding the pressure gradients.

\section{Numerical phenomenological model}

\section{A. Modelling of the plasma actuator}

The mathematical model used in this study to model the effects of plasma actuators was developed by 1 and further developed and improved in 2. It provides a body force distribution from the solution of a Laplace's equation for electric potential due to the voltage applied to the electrodes and a Poisson-like 2nd order partial differential equation for the charge density of the ionized working fluid. We have chosen this model because of its simplicity, but also for the fact that it takes a physical approach starting from the Lorentz force equation, and for its ability to mimic the time-averaged effect of real life DBD plasma actuators $(1,2,16,17)$.

The derivation starts with the assumption, that the magnetic force is negligible and the electrohydrodynamic (EHD) force can be expressed as:

$$
\vec{f}_{B}=\rho_{c} \vec{E}
$$

Where $\rho_{c}$ is the charge density and $\vec{E}$ is the electric field. Assuming further that the time variation of the magnetic field can be neglected, Maxwell's equation then states that the electric field $\vec{E}$ is conservative (i.e. $\nabla \times E=0)$, allowing it to be expressed in terms of the gradient of a scalar potential $(\vec{E}=-\nabla \Phi)$. Together with Gauss's law $\left(\nabla \cdot E=\rho / \varepsilon_{0}\right)$ this is combined to:

$$
\nabla \cdot(\varepsilon \nabla \Phi)=-\rho_{c}
$$


Assuming that the charge above the embedded electrode does not extend far (i.e. the Debye length is small, typically on the order of $10^{-4} \mathrm{~m}$ for gas discharge at atmospheric conditions) and that the gas particles are weakly ionized, the potential $\Phi$ is split into $\phi$, the electric potential due to the external electric field and $\varphi$, the electric potential due to the net charge density. Equation (3) is thus replaced by two separate equations, which also introduce the relative permittivity properties, $\varepsilon_{r}$, of the working fluid and plasma actuator materials:

$$
\begin{gathered}
\nabla \cdot\left(\varepsilon_{r} \nabla \phi\right)=0 \\
\nabla \cdot\left(\varepsilon_{r} \nabla \varphi\right)=-\left(\rho_{c} / \varepsilon_{0}\right)
\end{gathered}
$$

Using the equation describing the net charge density in a plasma and introducing the Debye length $\lambda_{d}$, a relationship between $\varphi$ and $\rho_{c}\left(\varphi=\left(-\rho_{c} \lambda_{d}^{2} / \varepsilon_{0}\right)\right)$ can be established (1). This allows Equation (5) to be recast into its final form as an equation for the charge density itself:

$$
\nabla \cdot\left(\varepsilon_{r} \nabla \rho_{c}\right)=\rho_{c} / \lambda_{d}^{2}
$$

Combining the results from Equation (4) and Equation (6) the body force can be computed from:

$$
\overrightarrow{f_{B}}=\rho_{c}(-\nabla \phi)
$$

Equation (4) and Equation (6) do not contain any time dependent terms and can be normalised by their respective maximum input values, $\phi^{\max }$, the maximum applied voltage and $\rho_{c}^{\max }$, the specified maximum charge density. Thus the equations only have to be solved once at the beginning of a simulation or externally. In order to obtain the instantaneous body force the normalised electric potential and charge density are multiplied by their respective maximum values as well as a wave from function $f(t)=\sin \left(2 \pi f_{A C} t\right)$, where $f_{A C}$ is the frequency of the $\mathrm{AC}$ voltage. With $\phi^{*}$ and $\rho^{*}$ denoting the normalised variables, the force can be computed from:

$$
\vec{f}_{B}(\mathbf{x}, t)=\left(\phi^{\max } f(t) \rho_{c}^{\max } f(t)\right) \rho_{c}^{*}(\mathbf{x})\left(-\nabla \phi^{*}(\mathbf{x})\right)
$$

The first and most frequently implemented version of the model (1) proposed to place, as a boundary condition for the charge density equation, a half Gaussian distribution starting at the corner of the embedded electrode closest to the exposed electrode. This introduces an additional parameter $(\sigma$, the standard deviation) controlling the behaviour/extent of the distribution above the embedded electrode. This approach was replaced by modelling the embedded electrode as a source of charge density instead (2). This reduces the number of input variables, improves the versatility of the model and its ability to be adapted for use in a $3 \mathrm{D}$ context.

Inputs into the model that affect the force distribution are the physical layout of the electrodes and dielectric, and their relative permittivities, as well as the Debye length $\lambda_{d}$. Other parameters such as $\phi^{\max }$, $\rho_{c}^{\max }$ and $f_{A C}$ will only affect the magnitude of the quantities and subsequently that of the body force, however not it's modelled distribution. All parameters but $\lambda_{d}$ and $\rho_{c}^{\max }$ are generally given, leaving the two as the only free inputs to be estimated. Both are physical quantities and can be varied within reason.

\section{B. Generation of the forcing term}

The model has been implemented in MATLAB using a standard 5-point (2D) and 7-point (3D) finite difference scheme, with sparse matrix storage, which allows for flexible actuator layouts and fast solving at high resolutions. The resulting matrices are symmetric and their solving can be accelerated with a Cholesky

pre-conditioner. For the 3D case a modified incomplete LU factorization preconditioned conjugate gradient solver is employed. The boundary conditions for the Laplace equation and for the Poisson-like equation are shown in figure 2. White is the air side of the computational domain, grey the dielectric material and dark-blue represents the electrodes (purple resembles the approximate body force distribution). In (2) no value is assigned to the exposed electrode in the computation of the charge density. However, it was found that to reproduce the same results, the electrode had to be prescribed as zero. Thus in all computations the electrodes are treated internal Dirichlet boundaries. In the computation of the charge density, the term on 
the right hand side is an absorption term, which depends on the Debye length and only acts on the air-side and is zero in the dielectric. Together with the zero valued boundaries, this results in charge density formation around entire length of the embedded electrode, which rapidly decreases in the normal direction away from the embedded electrode on the air-side, creating a thin layer of charge density. For thick dielectrics and spatially large force distributions, reproducing force distributions requires the use of large values for the Debye length. For a problem size of $2048^{2}$ the electric potential, charge density and body force can be computed in approximately $100 s$.
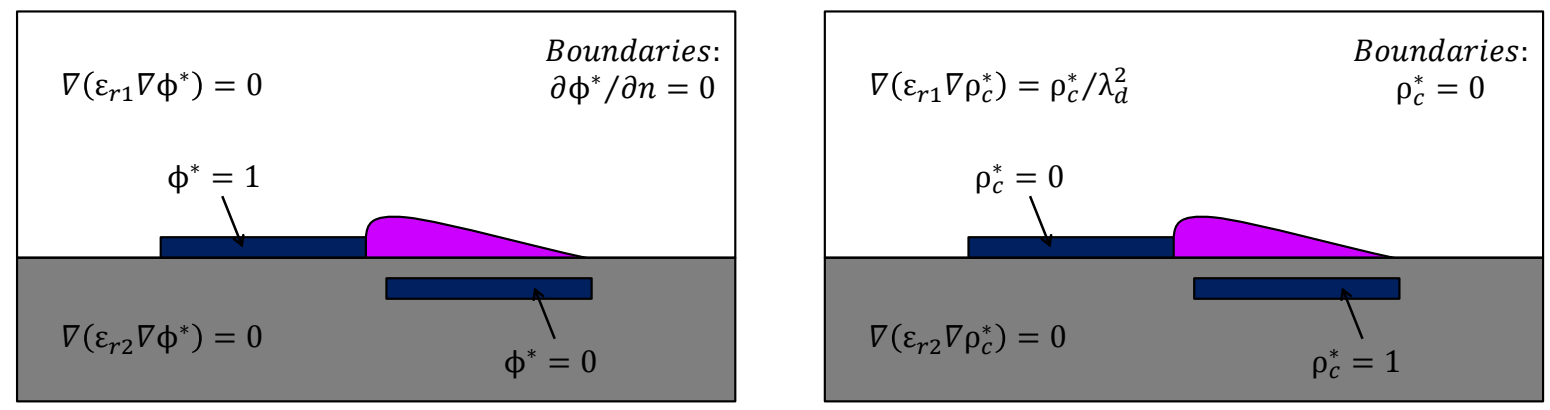

Figure 2. Boundary conditions for the Laplace's Equation for Electric Potential (left) and for the Poisson's Equation for Charge Density (right)

\section{Navier-Stokes solver for Direct Numerical Simulations (DNS)}

The forcing term generated with MATLAB to account for the effect of a DBD plasma actuator can be implemented in the high-order flow solver Incompact $3 \mathrm{~d}^{\mathrm{a}}$ as an extra forcing term in the incompressible Navier-Stokes equations. To solve those equations, finite-difference sixth-order compact schemes for spatial discretisation on a Cartesian mesh are used along with a third-order Adams-Bashforth scheme for time advancement. To treat the incompressibility condition, a fractional step method is required to solve a Poisson equation. For efficiency reasons, this equation is solved in spectral space using appropriate 3D Fast Fourier Transforms and with modified wave numbers in order to have a strict equivalence between all the operators. Note that the divergence free condition is ensured up to machine accuracy. When needed, a stretched mesh can be used in one direction in order to capture the small-scale dynamics with high fidelity. More details about the present code and its validation, especially the original treatment of the pressure in spectral space, can be found in 35. For all the simulations presented in this study, Inflow/Outflow boundary conditions are imposed in the streamwise direction ( $x$-direction), zero-velocity boundary conditions are imposed in the vertical direction ( $y$-direction) at the bottom of the computational domain while homogeneous Neumann boundary conditions are impose at the top of the domain.

\section{Results}

\section{A. Validation of the phenomenological model}

In order to validate the implementation of the plasma phenomenological model, the results for the configuration presented in (2) are reproduced and compared qualitatively. Table 1 summarises the configuration parameters.

Table 1. Parameters of the DBD plasma actuator used in the validation investigations.

\begin{tabular}{rrrrrr}
$\varepsilon_{r 1}$ & $\varepsilon_{r 2}$ & $\phi^{\max }$ & $f_{A C}$ & $\lambda_{d}$ & $\rho^{\max }$ \\
\hline- & - & $k V$ & $k H z$ & $m m$ & $C / m^{3}$ \\
\hline 1.0 & 2.7 & 5 & 4.5 & 0.17 & $7.5 \times 10^{-3}$
\end{tabular}

${ }^{\mathrm{a}}$ see www.incompact3d.com. 
Figures 3(a) and 3(b) show electrical potential and charge density distributions as well as the resulting normalised body force 3(c), which are in good agreement with the figures found in 2. Important points of reference are the extent of the normalised charge density in the $y$-direction above the embedded electrode (determined by the choice of Debye length), the location of the peak body force above the corner of the embedded electrode closest to the exposed electrode and the rate of decrease of force along the embedded electrode. It should be noted that the Suzen \& Huang model has been designed for low amplitude voltage with a relatively small extend for the body force (few millimetres). The length obtained in figure 3 is in agreement with the top-view visualizations of the plasma discharge conducted in 36. A wider body force distribution can be obtained if the applied voltage peak amplitude is increased as we will see in the following sections.

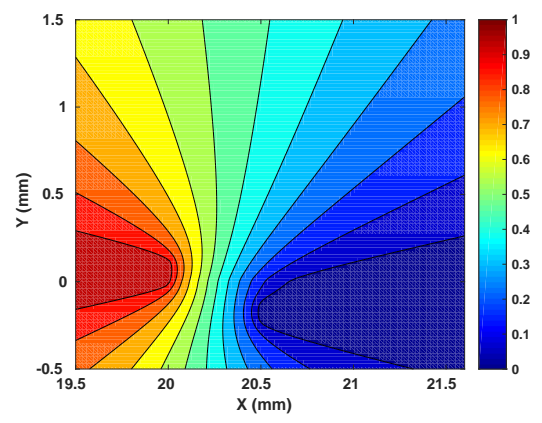

(a) Electric Potential

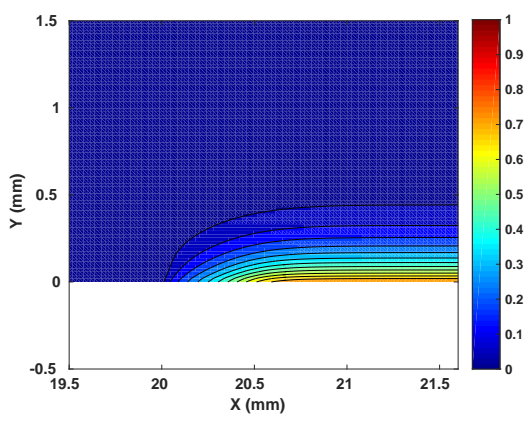

(b) Charge Density

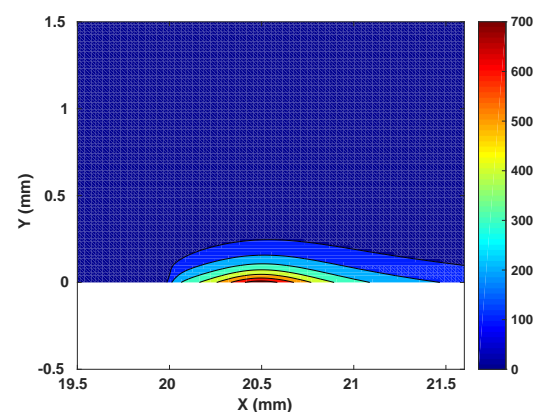

(c) Body Force Magnitude

Figure 3. Normalized Electric Potential $\phi / \phi^{\max }$, Charge Density $\rho_{c} / \rho_{c}^{\max }$ and Body Force Magnitude $\left|F_{b}\right| / \phi^{\max } / \rho_{c}^{\max }$ for our implementation of the Suzen \& Huang model.

\section{B. The starting vortex}

In order to validate our implementation of the Suzen \& Huang model in Incompact3d, we simulate the starting vortex generated by an asymmetric DBD plasma actuator in quiescent air and compare our results with the experimental data of $(31,32)$. Reproducing the main features of the experimental starting vortex will be a nice way to evaluate the robustness of the phenomenological Suzen \& Huang model. All the details about the experimental set-up can be found in $(31,32)$. The DBD plasma actuator used in these experiments was photochemically etched from a copper-clad Mylar sheet $(250 \mu m$ thick, dielectric constant $\varepsilon=3.1)$ and had $17 \mu \mathrm{m}$ thick upper and lower electrodes with widths of $2.5 \mathrm{~mm}$ and $6 \mathrm{~mm}$, respectively. The total length of the actuator was $160 \mathrm{~mm}$. For this comparison, we focus on one particular case for which the plasma is formed by applying sinusoidal AC waveforms with $\phi^{\max }=5.50 \mathrm{kV}$ at a frequency of $20 \mathrm{kHz}$ (Case A in 32).

The plasma forcing terms are illustrated in figure 4. It can be noted that for this particular set up, the resulting force from the DBD plasma actuator is very intense and very localized close to the electrode. The spatial distribution of the wall-parallel component is limited to the region close to the air-exposed electrode while the wall-normal component is distributed over a longer streamwise distance. The amplitude of both components is quite large and the use of a model taking into account only the wall-parallel component, as already done in few studies $(30,29)$, is debatable. In order to investigate the influence of the wallnormal component of the Suzen \& Huang model, two simulations, one with the wall-parallel and wallnormal components of Suzen \& Huang model, one without the wall-normal component, are performed with $n_{x} \times n_{y}=513 \times 257$ mesh nodes for a computational domain of size $L_{x} \times L_{y}=40 L \times 20 L$ where $L$ is the reference length equal to $1 \mathrm{~mm}$. The reference velocity $U_{\max }=0.45 \mathrm{~m} / \mathrm{s}$ is the maximum velocity obtained in the experiments of 32 for Case A. In order to reach $U_{\max }=0.45 \mathrm{~m} / \mathrm{s}$, the Reynolds number in our simulations is equal to 67 . All the quantities with no units in this study are made dimensionless using $L$ and $U_{\max }$. The computational domain is stretched in the $y$ - direction in order to have a very good accuracy near the wall. $\Delta x$ is equal to 0.078125 , the smallest $\Delta y$ is equal to $2.25 \times 10^{-2}$ and the largest $\Delta y$ is equal to 0.27 and the time step is equal to 0.00125 .

Figure 5 shows a series of flow visualisations (extracted from 32), PIV vorticity fields (extracted from 31) and vorticity fields from the present simulations with and without the wall-normal component of the Suzen $\&$ Huang model at three different times. The plasma initiates at $(x, y)=0$, the end of the upper electrode 

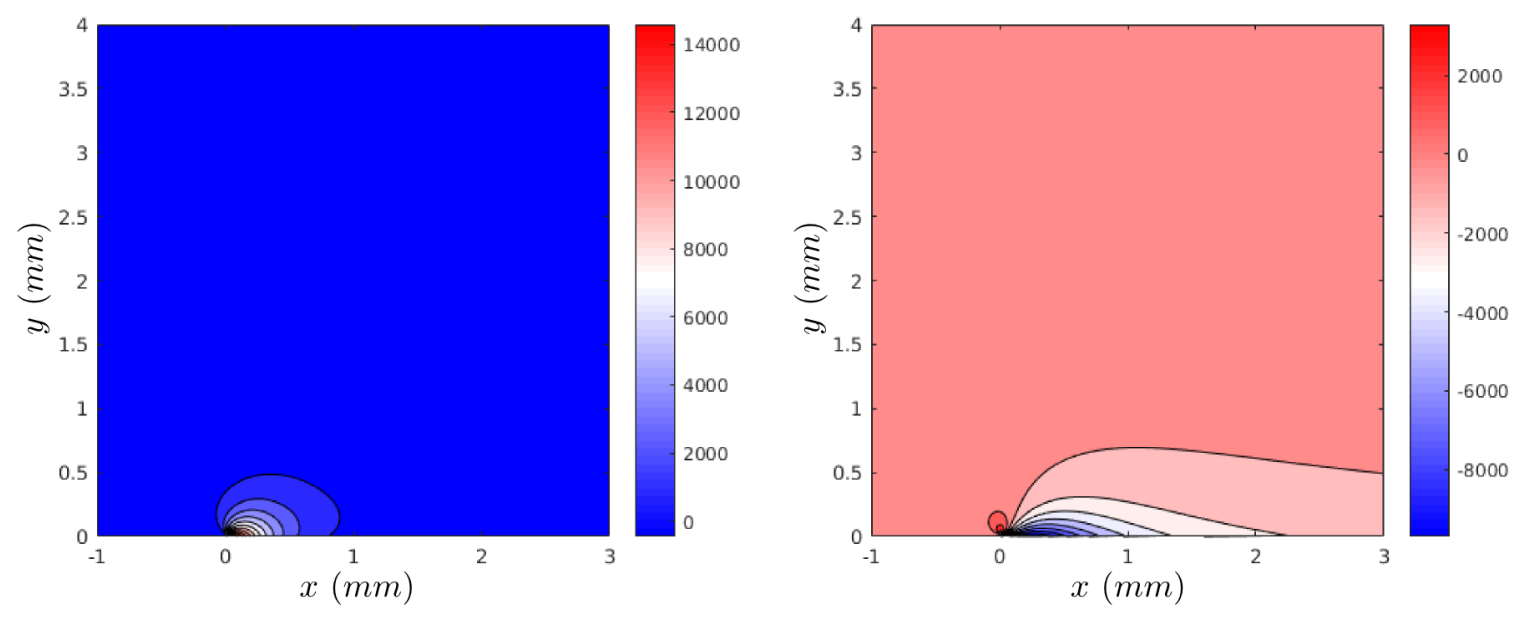

Figure 4. Plasma forcing term for the simulations: wall-parallel component (left) and wall-normal component (right). Force in $\mathrm{N} / \mathrm{m}^{3}$.

with $y=0 \mathrm{~mm}$ being the location of the wall. The plasma extends for around $2-3 \mathrm{~mm}$ in the horizontal direction (to the right). It can be seen that very quickly a single starting vortex is created. It is possible to see the number of turns very clearly with the smoke visualisations (first column of figure 5) as a result of entrainment of the ambient fluid into the vortex. The core of the vortex is moving along the positive $x$-direction and away from the wall in the positive $y$-direction. Our implementation of the Susan \& Huang model seems to be able to reproduce the formation and evolution of the starting vortex. It should be noted that the shape and location of the starting vortex is slightly different when the wall-normal component of the model is remove (fourth column of figure 5). In order to better quantify the quality of the Suzen \& Huang model, it is possible to track the evolution of the core of the vortex and to compare it with the experimental data.

The temporal evolution of the vortex core is presented in figure 6 for our two simulations. $x_{c}$ and $y_{c}$ are the coordinates of the vortex core. As suggested by 32, the data for $x_{c}$ and $y_{c}$ can be collapsed to straight lines when plotted in loglog axes. The development of the vortex core can be represented by a power law of the form

$$
x_{c}=\kappa_{1} t^{q} \quad y_{c}=\kappa_{2} t^{p} .
$$

Here, $\kappa_{1}=0.43, \kappa_{2}=0.95, q=0.64$ and $p=0.54$ for the simulation with the two components of the Suzen \& Huang model. These values have been obtained with a conventional non-linear least-squares (NLLS) Marquardt-Levenberg algorithm to fit our data. Hence, we can conclude that the starting vortex travels at an angle of about $32.5^{\circ}$ with the wall, given by $\arctan \left(y_{c} / x_{c}\right)$. This is in very good agreement with the experimental observations of 32 where an angle of $31^{\circ}$ was reported. It should be noted however that the exponents $q$ and $p$ are slightly different in this simulation whereas they are the same in the experiments of 32. For the simulation without the wall-normal component of the Suzen \& Huang model, we get $\kappa_{1}=0.47$, $\kappa_{2}=0.8, q=0.64$ and $p=0.5$, leading to a starting vortex travelling at an angle of about $43^{\circ}$. It is clear that for this particular benchmark (Case A in 32), the wall-normal component of the Suzen \& Huang model cannot be neglected. This component seems to have a strong impact of the vertical evolution of the starting vortex and is crucial for an accurate representation of the effect of a DBD plasma actuator.

\section{Comparisons between the PIV-based model and the Suzen \& Huang model}

In order to investigate the relevance of the Suzen \& Huang model, we need to compare it with our forcing term obtained experimentally via the Navier-Stokes approach. Initially, the Suzen \& Huang model has been designed for reproducing the body force developing on a thin dielectric layer where the applied peak amplitude voltage and frequency are low and high, respectively (by comparison with actuators with a thicker dielectric barrier). Here, the Suzen \& Huang model (referenced as S\&H in the figures) is tested for the modelling of a DBD plasma actuator with a thick dielectric layer with specific operating conditions to be imposed. The parameters for the present investigation are given in table 2 and the resulting time-averaged components 

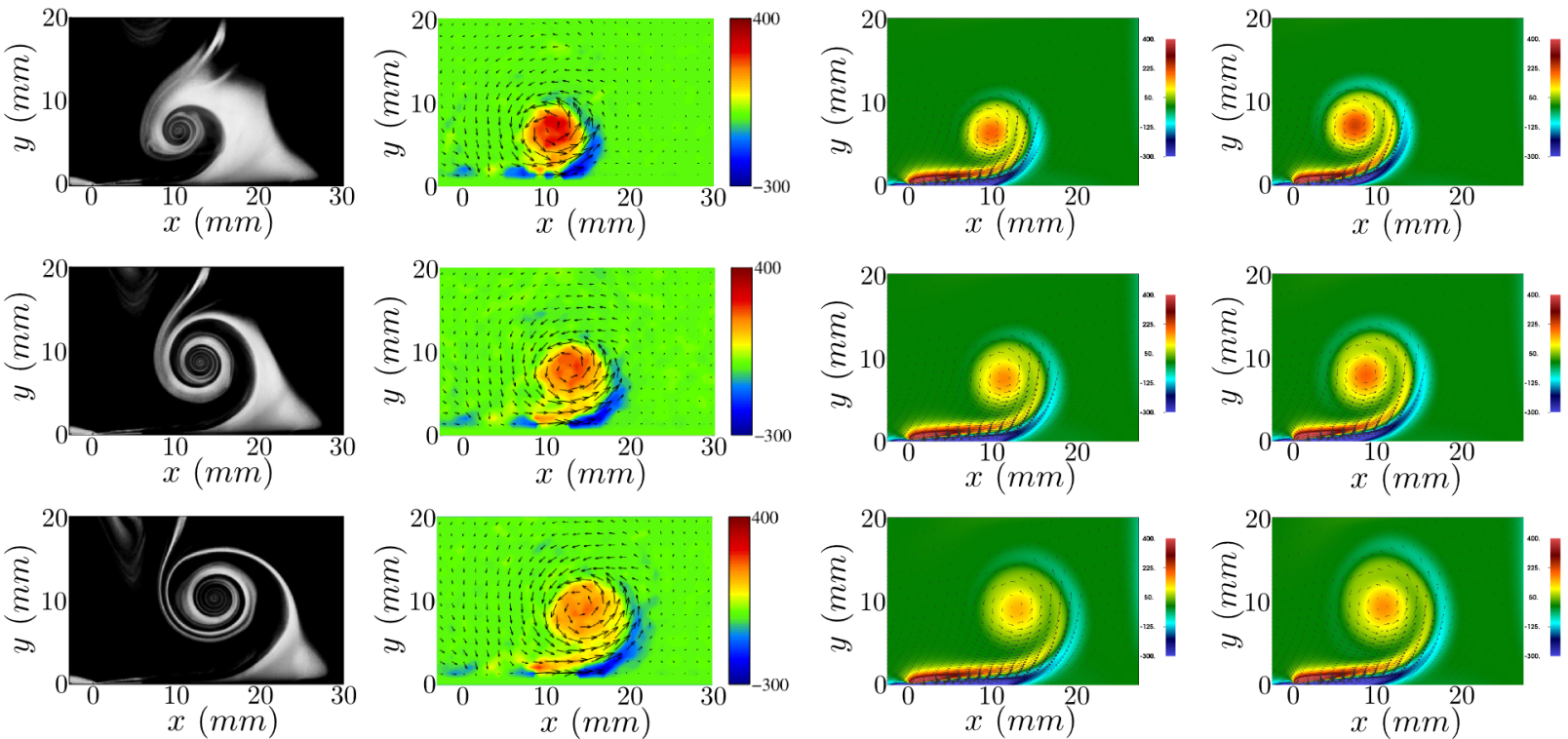

Figure 5. Flow visualisations (left column, extracted from 32), PIV vorticity fields (second column, extracted from 31, numerical vorticity fields with the two components of the Suzen \& Huang model (third column) and vorticity fields without the wall-normal component of the Suzen \& Huang model (fourth column) at $T=120,160$ and $200 \mathrm{~ms}$ from top to bottom. The unit for the vorticity fields is $\mathrm{s}^{-1}$.
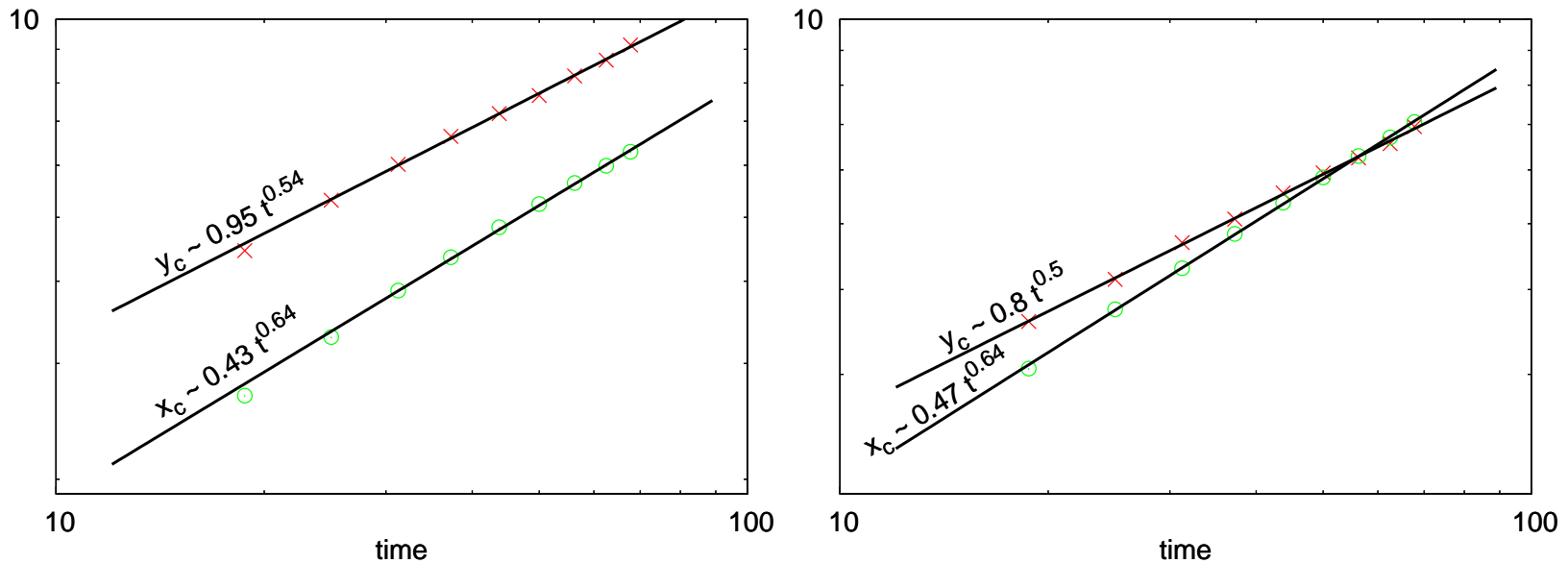

Figure 6. Vortex core scaling for the simulation with the two components of the Suzen \& Huang model (left) and without the wall-normal component of the Suzen \& Huang model (right).

Table 2. Parameters for the DBD plasma used in the experimental/numerical investigations.

\begin{tabular}{rrrrrr}
$\varepsilon_{r 1}$ & $\varepsilon_{r 2}$ & $\phi^{\max }$ & $f_{A C}$ & $\lambda_{d}$ & $\rho^{\max }$ \\
\hline- & - & $k V$ & $k H z$ & $m m$ & $C / m^{3}$ \\
\hline 1.0 & 4.0 & 20 & 1.0 & 2.5 & $2.0 \times 10^{-3}$
\end{tabular}


of the volume force can be seen in figure 7 (middle). At first, it seems that the numerical model is not able to capture the finest features of the experimental model as seen in figure 7 (top), especially for the wall-normal component. Major discrepancies can be observed close to the electrodes but also further away, between 5 and $30 \mathrm{~mm}$. In particular, it seems that the wall-normal component of the Suzen \& Huang model is much more intense and more extended than its experimental counter-part. This can be related to the two-dimensional assumptions used in the numerical model and the charge density boundary condition. It was indeed shown very recently in 8 (see Figure 15) that for three-dimensional simulations, with a nonuniform body force in the spanwise direction, the intensity and extend of the wall-normal component of the volume force can be reduced substantially by comparison to two-dimensional simulations. Phenomenological and first principles models are usually two-dimensional, meaning that both glow and streamer regimes are supposed to develop in a same plan. The homogeneity of the glow regime along the span of the air-exposed electrode is a well recognized phenomena. However, it is also widely admitted that streamers develops at stochastic locations along the air-exposed electrode. For PIV-based models such as the one proposed in this study, the occurrence of streamers in the measurement plane is not guaranteed. Because the wall-normal component the Suzen \& Huang model largely spreads over the dielectric barrier, we could think that this component is intimately connected with the propagation of the streamers. However, it is just a supposition, not an assumption. Further investigations would be needed to clarify this point. Concerning the wall-parallel component, the numerical and experimental data are in fairly good agreement with however a reduced shaped for the numerical model. Note finally that in various previous applications of the Suzen \& Huang model its wall-normal component is either not mentioned or assumed negligible $(16,37,38)$. However, first principle investigations by 39 indicate that the wall-normal component thrust could be on the same order as the wall-parallel component. This point will be discussed later.
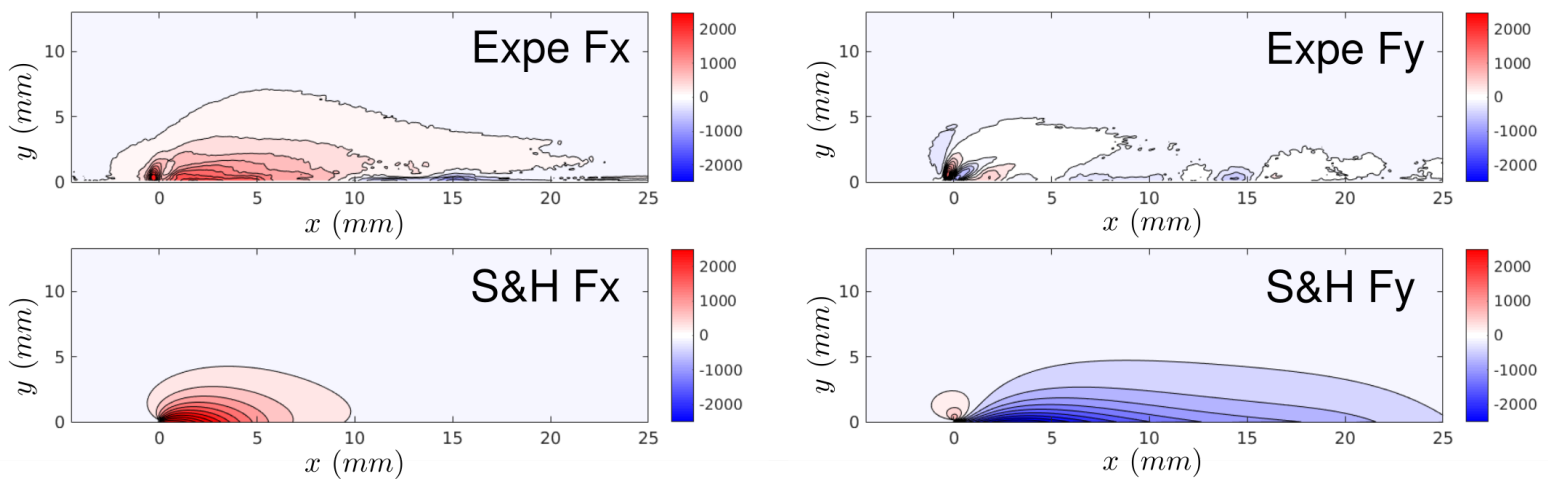

Figure 7. Spatial distribution of the wall-parallel (left) and wall-normal (right) components of the forcing term from the experimental data (top), from the Suzen \& Huang model (bottom).

Different flow fields are generated and compared with the reference ones obtained by our PIV measurements. Four simulations are performed, two with the experimental forcing (with and without the wallnormal component) and two with the Susan \& Huang model (with and without the wall-normal component). The simulations are performed with $n_{x} \times n_{y}=1537 \times 385$ mesh nodes for a computational domain $L_{x} \times L_{y}=240 L \times 60 L$ where $L$ is the reference length equal to $1 \mathrm{~mm}$. The simulations are performed on a single core on a powerful workstation and the wall-clock time for each simulations is only few hours. The reference velocity $U_{\max }=4.5 \mathrm{~m} / \mathrm{s}$ is the maximum velocity measured in our PIV experiments (33). The Reynolds number based on $L$ and on $U_{\max }$ is equal to 336 in our simulations. All the quantities with no units in this section are made dimensionless using $L$ and $U_{\max }$. The computational domain is stretched in the $y$ - direction in order to have a very good accuracy near the wall. $\Delta x$ is equal to 0.15625 , the smallest $\Delta y$ is equal to $5.12 \times 10^{-2}$ and the largest $\Delta y$ is equal to 0.477 and the time step is equal to 0.005 .

We first compare the time-average flow field obtained in the surrounding of the plasma actuators. Figure 8 (left) shows 2D maps of the time-averaged streamwise velocity component for the reference PIV data and the four simulations. The velocities are normalized with $U_{\max }$ so that all the values are between 0 (in blue) and 1 (in red). As expected a wall-jet flow parallel to the wall is obtained very close to the wall for $(y \approx 1 \mathrm{~mm})$. The simulations are in fairly good agreement with the experimental results. In particular, the simulations for which the wall-normal component is present are producing very similar results, at least qualitatively. The 

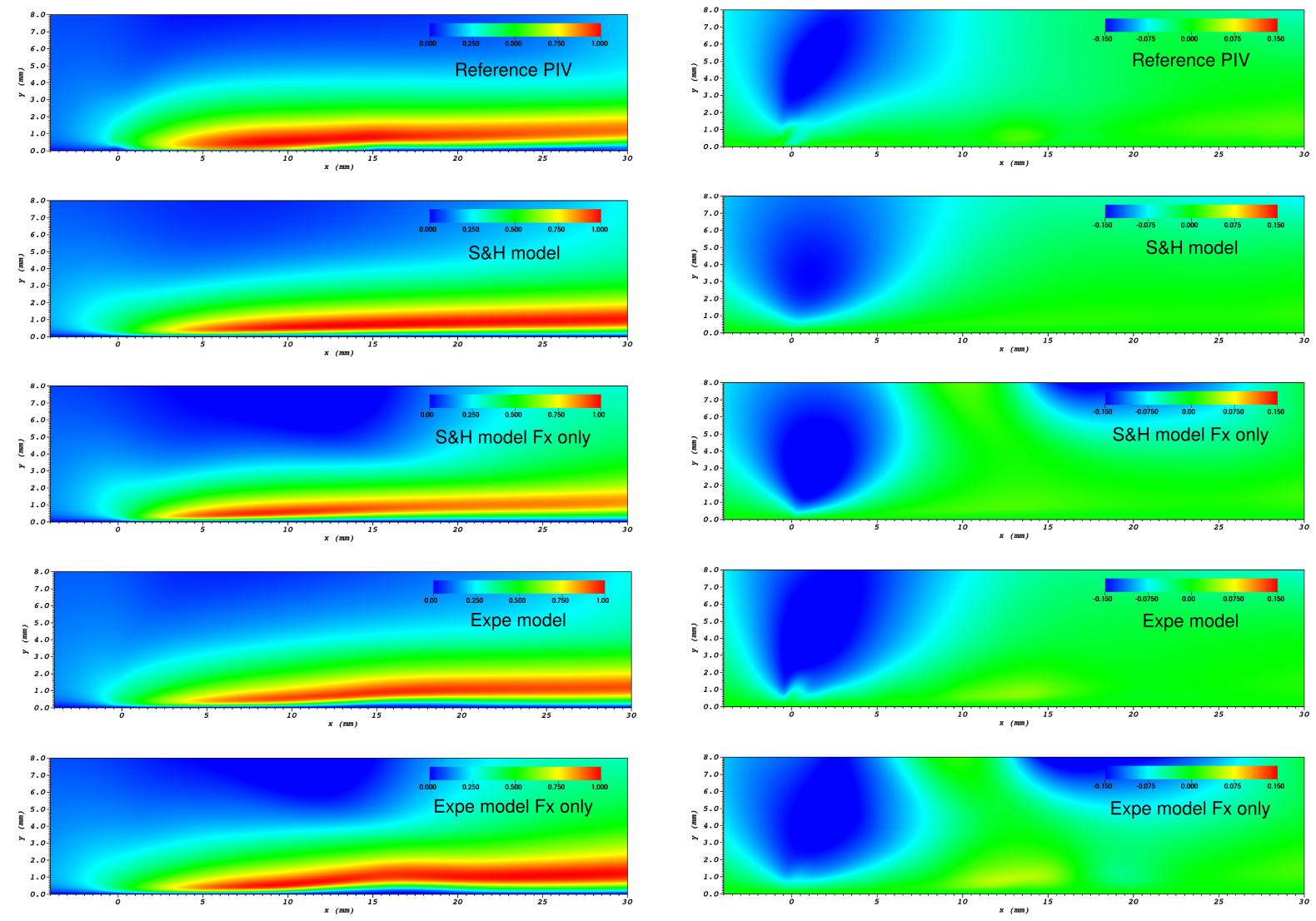

Figure 8. 2D maps of time-averaged streamwise velocity component (left) and vertical velocity component (right) normalized by $U_{\max }$ with from top to bottom: reference PIV data, simulation with the Suzen \& Huang model (with and without the wall-normal component) and with the experimental model (with and without the wall-normal component). 
main difference between the PIV data and the simulations is that both models are not able to reproduce the flow physics between 5 and $10 \mathrm{~mm}$ at the wall. These discrepancies in the near-wall region can also be attributed to a slight error in the exact localization of the dielectric wall in the experiments. Indeed, the resolution of the experimental data is very fine (about $8 \mu \mathrm{m}$ per $\mathrm{mm}$ ) but due to manufacturing constraint, a PMMA dielectric plate has not a strictly constant thickness $(3 \mathrm{~mm} \pm 0.4)$, making it complicated to locate with great accuracy the zero position. In the future, these two issues would need to be addressed. The simulations without the wall-normal component are evidence that the latter cannot be neglected. For the Suzen \& Huang model, we can observe a substantial reduction in the intensity of the wall-jet whereas for the experimental model we can observe a region where the flow separate at the wall (between 7.5 and $20 \mathrm{~mm}$ ). This flow separation originates from the small region with a negative wall-parallel volume force observed in the experiments at $x=10-17 \mathrm{~mm}$ as seen in figure 7 . As a consequence, the wall-jet is not parallel to the wall in this area. Also, the time-averaged streamwise velocity component is very close to zero for $y>5 \mathrm{~mm}$ and for $x>20 \mathrm{~mm}$ in the PIV data and in the simulations with the two components of the models whereas it is close to 0.5 for the simulations without the wall-normal components of the models.

Figure 8 (right) shows 2D maps of the time-averaged vertical velocity component for the reference PIV data and for the four simulations. The values are normalized with $U_{\max }$. Once again, it is clear that when the wall-normal component of the models is removed the results are inaccurate especially downstream of the plasma discharge that physically propagates up to $x \approx 10 \mathrm{~mm}$ (40). It is particularly visible in figure 8 (right) with an unexpected area of negative values for the time-averaged vertical velocity for $x>20 \mathrm{~mm}$ and $y>5 \mathrm{~mm}$ both for the experimental model and the Suzen \& Huang model without the wall-normal component. The appearance of this area of negative values is not fully understood at this point. The velocity profiles in figure 9 show that when the wall-normal component is removed from the models then the streamwise velocity component do not have zero values away from the wall, as observed in the PIV velocity profiles. It seems to indicate that the wall-normal component of the models (negative force toward the wall) is somehow limiting the effect of the wall-parallel component to a region close to the wall, leaving the flow undisturbed away from the wall.

The negative area above the electrode (see the vertical velocity component around $x=0 \mathrm{~mm}$ ) is reduced when the wall-normal component is not present. For the simulations with both components of the model, the main difference between the experimental model and the Suzen \& Huang model is located between 5 and $15 \mathrm{~mm}$ with a small area of positive values for the experimental model. This area is not present for the Suzen \& Huang model nor in the reference PIV data. The bottom of the negative area above the electrode (around $x=0 \mathrm{~mm}$ and $y=1 \mathrm{~mm}$ ) seems to be closer to the experimental PIV data for the simulation with the experimental model. Also, the suction area above the electrodes is slightly tilted to the right for the PIV data and for the simulation with the experimental forcing whereas it is not the case for the simulation with the Suzen \& Huang model. A slight inclination of the experimental set-up cannot be excluded at this point and further investigations will be needed to elucidate this point. Overall, it can be concluded that the Suzen \& Huang model would need to be improved for a more accurate reproduction of the PIV data. One has to recognize that the results obtained with this phenomenological approach gives unexpected good results considering the fact that it has been designed for a different range of parameters in 1 (low amplitude voltage with a relatively small extend for the body force).

In order to better investigate the quality of our simulations, we plot in figure 9 vertical profiles of the dimensional time-averaged streamwise velocity component at different streamwise locations from $x=2.5 \mathrm{~mm}$ to $x=25 \mathrm{~mm}$. At $x=2.5 \mathrm{~mm}$ and $x=5 \mathrm{~mm}$, only the simulation with the Suzen \& Huang model without the wall-normal component is able to reach the maximum velocity of the PIV-data however the thickness of the wall-normal jet is incorrect. Actually, at these two locations, none of the simulations are able to achieve the correct thickness for the wall-normal jet. Interestingly, further away from the actuator for $x>15 \mathrm{~mm}$, the profiles obtained with the simulation based on the experimental model seems to fit nicely with the PIVdata profiles close to the wall (up to $y=3-4 \mathrm{~mm}$ ). The influence of the wall-normal component can clearly be seen at $x=10 \mathrm{~mm}$ and at $x=25 \mathrm{~mm}$ away from the wall. When the wall-normal component is not present, the simulations are not able to fit the PIV-data profiles for $y>3-4 \mathrm{~mm}$ with quite small values (50\% smaller than the PIV-data ones) at $x=10 \mathrm{~mm}$ and with quite large values (50\% larger than the PIV-data ones) at $x=25 \mathrm{~mm}$. It is another evidence that the wall-normal component cannot be neglected for a better reproduction of the experimental results but needs to be improved for a better fit with the reference PIV data.

In order to check the assumption that the pressure gradients can be neglected in the PIV-based approach, 

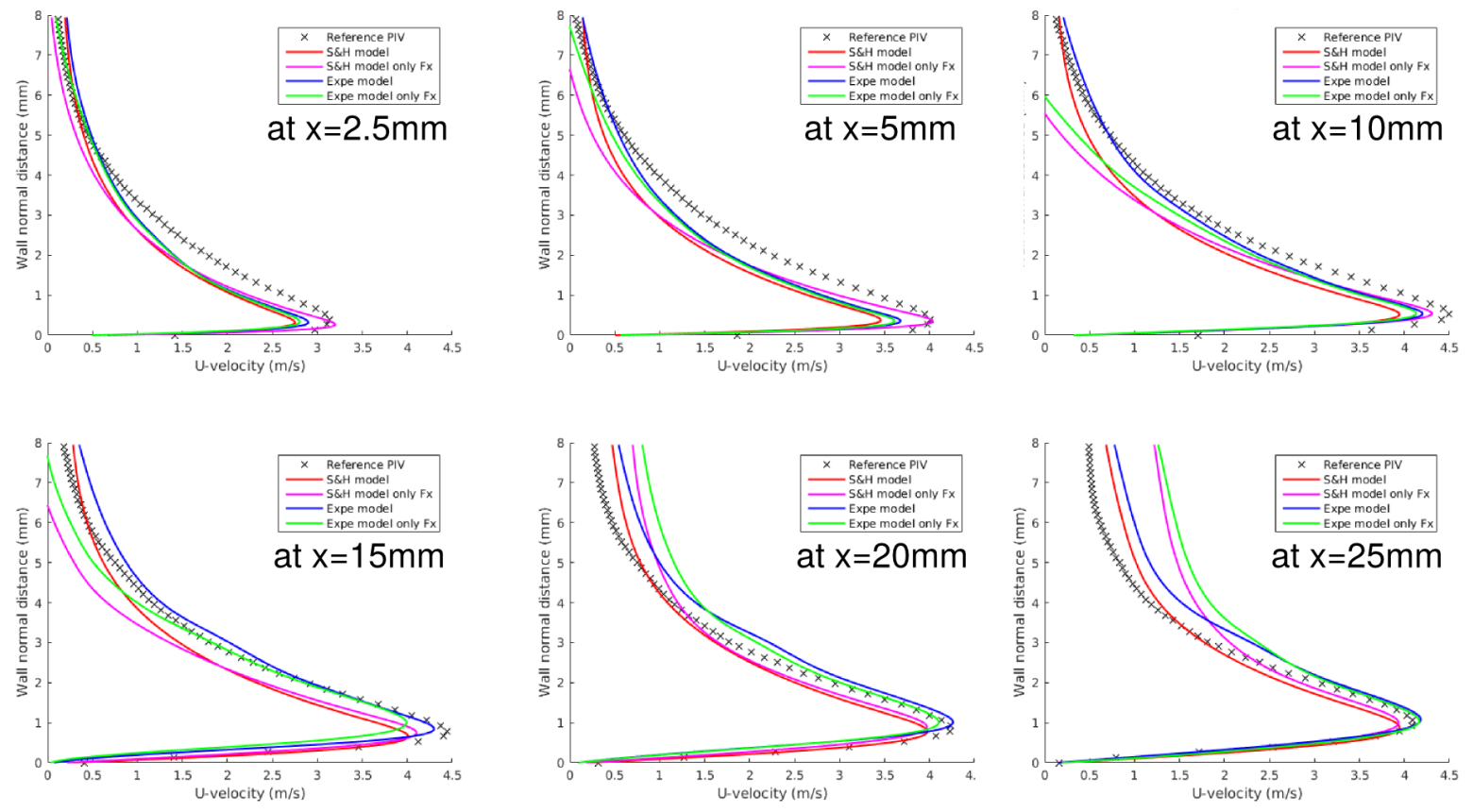

Figure 9. Vertical profiles of the dimensional time-averaged streamwise velocity component at 6 different streamwise locations from $x=2.5 \mathrm{~mm}$ to $x=25 \mathrm{~mm}$.

we plot in figure 10 the time-averaged pressure gradients obtained in our simulation with the Suzen \& Huang model and compare it with the actual wall-parallel and wall-normal forcing terms. The first important result is that shape of the pressure gradients obtained here is similar to the one obtained in 29 (see Figures 4 and 6). A crucial observation is that the values obtained for the pressure gradients are actually of the same order as the values obtained for the wall-parallel and wall-normal forcing terms, indicating that the pressure gradients terms cannot be neglected in the Navier-Stokes approach for this particular set-up and for this particular DBD plasma actuator. It is important to point out that in a similar flow configuration but for different parameters for the DBD plasma actuator, 29 found that the maximum amplitude of the pressure gradient $\partial p / \partial x$ located at the upstream edge of the force distribution can reach values up to $10 \%$ of the maximum wall-parallel force. Our results seem to demonstrate that the pressure gradient in the vertical direction $\partial p / \partial y$ has a same similar shape as the wall-normal forcing term which suggests that if we remove the pressure gradients from the forcing term (to better match the PIV-based approach) it should reduced the intensity of the wall-normal forcing term. It can be seen in figure 11 (bottom, right) that it is indeed the case, confirming the important contribution of the pressure gradient in the PIV-based approach. Concerning the wall-parallel component minus the streamwise pressure gradient $\partial p / \partial x$, its shape has spread in the streamwise direction and it is now very similar to the wall-parallel component of the experimental forcing term as seen in figure 11 (bottom, left). The wall-parallel component of the experimental forcing exhibits a small area with weak negative values at the wall between $x=7.5 \mathrm{~mm}$ and $x=12.5 \mathrm{~mm}$ which is not present in the numerical data. It could be related to the difficulty to get accurate PIV data very close to the wall. The spatial distribution of the wall-normal component minored by the vertical pressure gradient $\partial p / \partial y$ also confirms that the PIV-based approach is not a strict modelling of the volume force caused by the discharge. For this component, a quite good agreement is found close to the air-exposed electrode. However, as it was postulated in 28 , the visual trace of a volume force beyond the plasma extension (i.e. $x>10 \mathrm{~mm}$ ) is fully caused by the pressure gradient $\partial p / \partial y$. The method proposed in this paper gives precious information regarding the discrimination between volume force and pressure gradients in the PIV-based model.

From these first investigations, it can be concluded that the influence of the pressure gradients needs to be investigated experimentally. It means that the PIV-based method has to be revisited in order to take into consideration the contribution of the pressure gradients. This requires the measurement of the pressure distribution in a plane while the plasma discharge is operated. It is a very challenging task and 

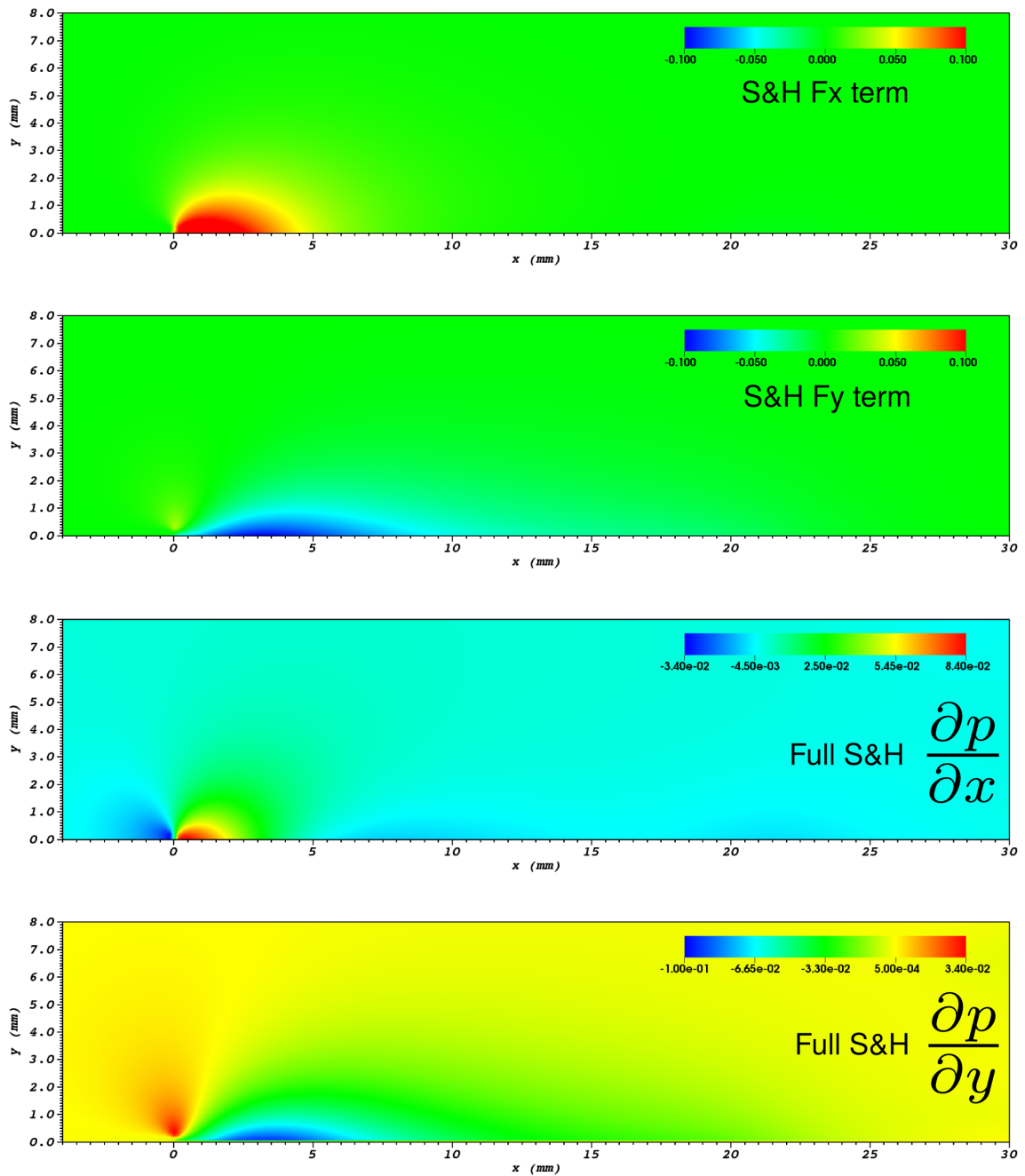

Figure 10. Wall-parallel forcing term from the Suzen \& Wang model (top) compared with the time-average pressure gradients $\partial p / \partial x$ (middle) and $\partial p / \partial y$ (bottom). Same parameters as in figure 7. 
up to now such experimental data are not available. Research focusing on the experimental determination of the pressure field and its gradients are mandatory. In absence of an external force, the local distribution of the pressure can be quite easily measured (41). Here, the pressure field should be evaluated by optical techniques such as Backward-Oriented Schlieren, a method in which the gas density gradient can be directly measured (providing a sufficient sensitivity of the measurement system) and thus the pressure can be roughly estimated. A coupling between PIV and BOS approaches may be a solution for improving the fidelity of the PIV-based models.
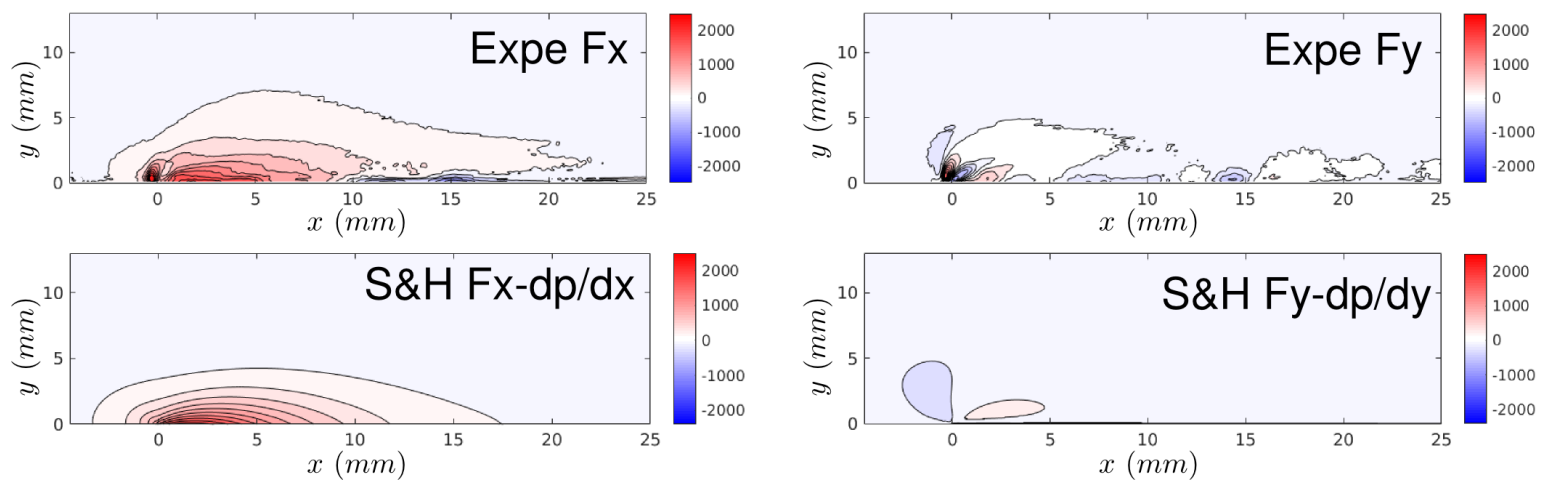

Figure 11. Spatial distribution of the wall-parallel (left) and wall-normal (right) components of the forcing term from the experimental data (top) and from the Suzen \& Huang model minus the time-average pressure gradients (bottom).

\section{Conclusion}

We performed in this study two-dimensional high-fidelity simulations of the flow induced by a DBD plasma actuator using an experimental volume force extracted from PIV measurements via a reverse NavierStokes approach and with the phenomenological Suzen \& Huang model. The main results can be summarized as follow:

- Using the phenomenological Suzen \& Huang model, we managed to reproduce the experimental results of 31 and 32 for the formation and evolution of a starting vortex in a quiescent fluid. In particular, the spatio-temporal evolution of the core of the starting vortex was in very good agreement with the experimental data.

- We highlighted the importance of the wall-normal component of the phenomenological Suzen \& Huang model. This component cannot be neglected for a realistic reproduction of the effect of a DBD plasma actuator.

- Some differences between the phenomenological model and the experimental model have been observed and none of the models are able to predict the thickness of the wall-jet close to the actuator.

- The pressure gradients which are assumed to be very small by comparison to the components of the model in the experimental reverse Navier-Stokes approach are found to be of the same order as the component of the volume force.

- The experimental model extracted from PIV data via a reverse Navier-Stokes approach seems to be quite promising for the simulation of the flow induced by a DBD plasma actuator in a quiescent fluid, with a better match with the reference PIV data than the phenomenological Suzen \& Huang model. It is a puzzling observation as the pressure gradients are actually non negligible according to our simulations.

Further simulations are needed in order to better understand the influence of the pressure gradients close to the plasma actuator. In particular, we are planning to run a simulation with the phenomenological Suzen \& Huang model where the pressure gradients will be removed from the forcing term (as seen in 
figure 11, bottom). The idea is to mimic the experimental forcing term for a better understand of the flow physics. Some calibration will be needed for this simulation so that the global thrust of the new model matched the experimental one for both components (figure 7, top). Some work is also need in order to improve the phenomenological Suzen \& Huang predictions, in particular on the wall-normal component. For instance, one could limit the extent of the plasma over the embedded electrode in a manner analogous to the implementation of the first version of the Suzen \& Huang model (1) where a Gaussian distribution was used. The boundary condition prescribed to the embedded electrode for charge density in the present work is a constant value for the entire electrode. Experiments by 42 or by 26 however show that generally the plasma does not extend over the entire length of the embedded electrode. Finally, following the recent work of 7 and 8, we will investigate the phenomenological Suzen \& Huang model in a three-dimensional context with a non-uniform distribution of the volume forcing in the spanwise direction.

\section{Acknowledgements}

Timothy Brauner and Sylvain Laizet would like to thank EPSRC for their financial support (EP/M022676/1) and for the computational time made available on the UK supercomputing facility ARCHER via the UK Turbulence Consortium (EP/L000261/1). Nicolas Benard and Eric Moreau would like to thank the French ANR program and DGA under reference ANR-13-ASTR-0005 for the SINAPS project. A part of the experimental equipment used in this work was funded by the French Government program Investissements dAvenir (LABEX INTERACTIFS, reference ANR-11-LABX-0017-01). Finally, the authors would like to acknowledge the NATO Applied Vehicle Technology (AVT) 254 "Assessment of Plasma Actuator Technologies for Internal Flows".

\section{References}

\footnotetext{
${ }^{1}$ Suzen, Y., Huang, P., Jacob, J., and Ashpis, D., "Numerical simulations of plasma based flow control applications," AIAA paper 2005-4633, 2005.

${ }^{2}$ Suzen, Y., Huang, P., and Ashpis, D., "Numerical simulations of flow separation control in low-pressure turbines using plasma actuators," AIAA paper 2007-937, 2007.

${ }^{3}$ Moreau, E., "Airflow control by non-thermal plasma actuators," J. of Phys. D: Applied Physics, Vol. 40(3), 2007, pp. 605.

${ }^{4}$ Corke, T., Post, M., and Orlov, D., "SDBD plasma enhanced aerodynamics: concepts, optimization and applications," Progress in Aero. Sciences, Vol. 43(7), 2007, pp. 193-217.

${ }^{5}$ Cattafesta III, L. and Sheplak, M., "Actuators for active flow control," Ann. Rev. Fluid Mech., Vol. 43, 2011, pp. 247-272.

${ }^{6}$ Corke, T., Post, M., and Orlov, D., "Single dielectric barrier discharge plasma enhanced aerodynamics: physics, modeling and applications," Exp. Fluids, Vol. 46(1), 2009, pp. 1-26.

${ }^{7}$ Nishida, H., Nonomura, T., and Abe, T., "Three-dimensional simulations of discharge plasma evolution on a dielectric barrier discharge plasma actuator," J. of Applied Phys., Vol. 115(13), 2014, pp. 133301.

${ }^{8}$ Nishida, H., Nonomura, T., and Abe, T., "Numerical Study on Spanwise Nonuniformity in Body-Force Field of DielectricBarrier-Discharge Plasma Actuator," AIAA Journal, Vol. 54(2), 2016, pp. 659-669.

${ }^{9}$ Boeuf, J., Lagmich, Y., Unfer, T., Callegari, T., and Pitchford, L., "Electrohydrodynamic force in dielectric barrier discharge plasma actuators," J. of Phys. D: Applied Physics, Vol. 40(3), 2007, pp. 652.

${ }^{10}$ Singh, K. P. and Roy, S., "Force approximation for a plasma actuator operating in atmospheric air," J. of Applied Phys., Vol. 103(1), 2008, pp. 013305.

${ }^{11}$ Rogier, F., Dufour, G., and Kourtzanidis, K., "Numerical Simulation of sinusoidal driven DBD actuators and Comparisons with Experiments," AIAA paper 2014-2808, 2014.

${ }^{12}$ Parent, B., Shneider, M., and Macheret, S., "Detailed Modeling of Plasmas for Computational Aerodynamics," AIAA Journal, Vol. 54(1), 2016, pp. 1-14.

${ }^{13}$ Mertz, B. and Corke, T., "Single-dielectric barrier discharge plasma actuator modelling and validation," J. Fluid Mech., Vol. 669, 2011, pp. 557-583.

${ }^{14}$ Orlov, D., Corke, T., and Patel, M., "Electric circuit model for aerodynamic plasma actuator," AIAA paper 2006-1206, 2006.

${ }^{15}$ Shyy, W., Jayaraman, B., and Andersson, A., "Modeling of glow discharge-induced fluid dynamics," J. of Applied Phys., Vol. 92(11), 2002, pp. 6434-6443.

${ }^{16}$ Ibrahim, I. and Skote, M., "Simulating plasma actuators in a channel flow configuration by utilizing the modified SuzenHuang model," Computers and Fluids, Vol. 99, 2014, pp. 144-155.

${ }^{17}$ Eltaweel, A., Wang, M., Kim, D., Thomas, F., and Kozlov, A., "Numerical investigation of tandem-cylinder noise reduction using plasma-based flow control," J. Fluid Mech., Vol. 756, 2014, pp. 422-451.

${ }^{18} \mathrm{Li}, \mathrm{Z}$., Hu, B., Lan, S., Zhang, J., and Huang, J., "Control of turbulent channel flow using a plasma-based body force," Computers and Fluids, Vol. 119, 2015, pp. 26-36.
} 
${ }^{19}$ Rizzetta, D. and Visbal, M., "Numerical investigation of plasma-based control for low-Reynolds-number airfoil flows," AIAA journal, Vol. 49(2), 2011, pp. 411-425.

${ }^{20}$ Aono, H., Sekimoto, S., Sato, M., Yakeno, A., Nonomura, T., and Fujii, K., "Computational and experimental analysis of flow structures induced by a plasma actuator with burst modulations in quiescent air," Mech. Engineering J., Vol. 2(4), 2015, pp. 15-00233.

${ }^{21}$ Sato, M., Nonomura, T., Okada, K., Asada, K., Aono, H., Yakeno, A., Abe, Y., and Fujii, K., "Mechanisms for laminar separated-flow control using dielectric-barrier-discharge plasma actuator at low Reynolds number," Phys. Fluids, Vol. 27(11), 2015, pp. 117101.

${ }^{22}$ Asada, K., Nonomura, T., Aono, H., Sato, M., Okada, K., and Fujii, K., "LES of transient flows controlled by DBD plasma actuator over a stalled airfoil," Int. J. of Comp. Fluid Dynamics, Vol. 29(2-5), 2015, pp. 215-229.

${ }^{23}$ Albrecht, T., Weier, T., Gerbeth, G., Metzkes, H., and Stiller, J., "A method to estimate the planar, instantaneous body force distribution from velocity field measurements," Phys. Fluids, Vol. 23(2), 2011, pp. 021702.

${ }^{24}$ Kotsonis, M., Ghaemi, S., Veldhuis, L., and Scarano, F., "Measurement of the body force field of plasma actuators," J. of Phys. D: Applied Physics, Vol. 44(4), 2011, pp. 045204.

${ }^{25}$ Murphy, J. and Lavoie, P., "Characterization of DBD plasma actuators via PIV measurements," AIAA paper 2013-346, 2013.

${ }^{26}$ Kriegseis, J., Schwarz, C., Tropea, C., and Grundmann, S., "Velocity-information-based force-term estimation of dielectric-barrier discharge plasma actuators," J. of Phys. D: Applied Physics, Vol. 46(5), 2013, pp. 055202.

${ }^{27}$ Benard, N. and Moreau, E., "Electrical and mechanical characteristics of surface AC dielectric barrier discharge plasma actuators applied to airflow control," Exp. Fluids, Vol. 55(11), 2014, pp. 1-43.

${ }^{28}$ Benard, N., Debien, A., and E. Moreau, E., "Time-dependent volume force produced by a non-thermal plasma actuator from experimental velocity field," J. of Phys. D: Applied Physics, Vol. 46(24), 2013, pp. 245201.

${ }^{29}$ Dörr, P. and Kloker, M., "Numerical investigation of plasma-actuator force-term estimations from flow experiments," $J$. of Phys. D: Applied Physics, Vol. 48(39), 2015, pp. 395203.

${ }^{30}$ Maden, I., Maduta, R., Kriegseis, J., Jakirlić, S., Schwarz, C., Grundmann, S., and Tropea, C., "Experimental and computational study of the flow induced by a plasma actuator," Int. J. Heat and Fluid Flow, Vol. 41, 2013, pp. 80-89.

${ }^{31}$ Whalley, R., Turbulent boundary-layer control with DBD plasma actuators using spanwise travelling-wave technique, Ph.D. thesis, University of Nottingham, 2011.

${ }^{32}$ Whalley, R. and Choi, K., "The starting vortex in quiescent air induced by dielectric-barrier-discharge plasma," J. Fluid Mech., Vol. 703, 2012, pp. 192-203.

${ }^{33}$ Benard, N., Caron, M., and Moreau, E., "Evaluation of the time-resolved EHD force produced by a plasma actuator by particle image velocimetry-a parametric study," J. of Phys.: Conference Series, Vol. 646(1), 2015, pp. 12055-12058.

${ }^{34}$ Corke, T., Enloe, C., and Wilkinson, S., "Dielectric Barrier Discharge Plasma Actuators for Flow Control," Ann. Rev. Fluid Mech., Vol. 42, 2010, pp. 505-529.

${ }^{35}$ Laizet, S. and Lamballais, E., "High-order compact schemes for incompressible flows: a simple and efficient method with the quasi-spectral accuracy," J. Comp. Phys., Vol. 228(16), 2009, pp. 5989-6015.

${ }^{36}$ Kriegseis, J., Grundmann, S., and Tropea, C., "Power consumption, discharge capacitance and light emission as measures for thrust production of dielectric barrier discharge plasma actuators," J. of Applied Phys., Vol. 110(1), 2011, pp. 013305.

${ }^{37}$ Abdollahzadeh, M., Páscoa, J., and Oliveira, P., "Numerical modeling of boundary layer control using dielectric barrier discharge," MEFTE IV Conferencia Nacional em Mecanica de Fluidos, Termodinamica e Energia, 2012, p. 63.

${ }^{38}$ Asada, K., Nonomura, T., Aono, H., Sato, M., Okada, K., and Fujii, K., "LES of transient flows controlled by DBD plasma actuator over a stalled airfoil," Int. J. of Comp. Fluid Dynamics, Vol. 29(3-5), 2015, pp. 215-229.

${ }^{39}$ Unfer, T., Boeuf, J.-P., Rogier, F., and Thivet, F., "Modeling of dielectric barrier discharge and coupling with computational fluid dynamics," AIAA paper 2008-1375, 2008.

${ }^{40}$ Benard, N. and Moreau, E., "Role of the electric waveform supplying a dielectric barrier discharge plasma actuator," Applied Phys. Letters, Vol. 100(19), 2012, pp. 193503.

${ }^{41}$ van Oudheusden, B., Scarano, F., Roosenboom, E., Casimiri, E., and Souverein, L., "Evaluation of integral forces and pressure fields from planar velocimetry data for incompressible and compressible flows," Exp. in Fluids, Vol. 43(2-3), 2007, pp. $153-162$.

${ }^{42}$ Gregory, J., Enloe, C. L., Font, G., and McLaughlin, T., "Force production mechanisms of a Dielectric-barrier discharge plasma actuator," AIAA paper 2007-185, 2007. 